\title{
Representations on Hessenberg Varieties and Young's Rule
}

\author{
Nicholas Teff If $^{\dagger}$ \\ ${ }^{1}$ Department of Mathematics, University of Iowa, Iowa City, IA, USA
}

\begin{abstract}
We combinatorially construct the complex cohomology (equivariant and ordinary) of a family of algebraic varieties called regular semisimple Hessenberg varieties. This construction is purely in terms of the Bruhat order on the symmetric group. From this a representation of the symmetric group on the cohomology is defined. This representation generalizes work of Procesi, Stembridge and Tymoczko. Here a partial answer to an open question of Tymoczko is provided in our two main result. The first states, when the variety has multiple connected components, this representation is made up by inducing through a parabolic subgroup of the symmetric group. Using this, our second result obtains, for a special family of varieties, an explicit formula for this representation via Young's rule, giving the multiplicity of the irreducible representations in terms of the classical Kostka numbers.

Résumé. Nous construisons la cohomologie complexe (équivariante et ordinaire) d'une famille de variétés algébriques appelées variétés régulières semisimples de Hessenberg. Cette construction utilise exclusivement l'ordre de Bruhat sur le groupe symétrique, et on en déduit une représentation du groupe symétrique sur la cohomologie. Cette représentation généralise des résultats de Procesi, Stembridge et Tymoczko. Nous offrons ici une réponse partielle à une question de Tymoczko grâce à nos deux résultats principaux. Le premier déclare que lorsque la variété a plusieurs composantes connexes, cette représentation s'obtient par induction à travers un sous-groupe parabolique du groupe symétrique. Nous en déduisons notre deuxième résultat qui fournit, pour une famille spéciale de variétés, une formule explicite pour cette représentation par la règle de Young, et donne ainsi la multiplicité des représentations irréductibles en termes des nombres classiques de Kostka.
\end{abstract}

Keywords: Bruhat order, combinatorial representation theory, flag varieties, Young's rule

\section{Introduction}

In this paper we study a representation of the symmetric group on the complex cohomology (ordinary and equivariant) of a family of algebraic varieties called regular semisimple Hessenberg varieties. This representation exposes connections between the combinatorics of the symmetric group, the geometry of the varieties, and representation theory. Also, this representation generalizes representations in work of Procesi $[\mathbf{P}]$, Stembridge [St], and Tymoczko [T3]. Procesi and Stembridge studied this same representation in the case when the variety is the toric variety associated to the Coxeter complex in type $A_{n}$ using ordinary cohomology. Tymoczko studied it when the variety is the flag variety using equivariant cohomology.

\footnotetext{
${ }^{\dagger}$ Partially supported by the University of Iowa Department of Mathematics NSF VIGRE grant DMS-0602242 
These varieties are examples of regular semisimple Hessenberg varieties. In each case, a decomposition of the representation into irreducible representations are known. Here we partially answer an open question of Tymoczko [T3]. The question Tymoczko asks is; "can one obtain similar decompositions of this representation for the cohomology of all regular semisimple Hessenberg varieties?"

We answer this question in two cases. The first states, when the Hessenberg variety is disconnected, the representation is a particular induced representation through a parabolic (i.e. Young) subgroup [Theorem 4.10]. The second result provides an explicit irreducible decomposition of the representation for parabolic Hessenberg varieties [Definition 4.11] via Young's rule (see [JK] Chapter 2]). We give this decomposition in terms of classical Kostka numbers [Theorem 4.15].

Our approach is combinatorial. We study these varieties via a combinatorial graph called the moment graph. These graphs are subgraphs of the Bruhat graph for $S_{n}$ [BB, Chapter 2]. This allows us to use tools from Coxeter groups, for example the Bruhat order, parabolic subgroups, and minimal coset representatives. In fact, the results of this abstract can be extended to other Coxeter groups in other Lie types. We chose to remain in type $A$ to make the connection to combinatorics (e.g. partitions and Kostka numbers) more evident.

\subsection{Acknowledgments}

I would like thank Julianna Tymoczko for all of her assistance with the preparation of this paper, including help with the $\mathrm{LT}_{\mathrm{E}} \mathrm{X}$-code for moment graphs, Danilo Diedrichs for his French translation of the abstract, and the anonymous referees for their helpful comments.

\section{Hessenberg varieties.}

Fix $G=G L_{n}(\mathbb{C})$ and let $B$ be the subgroup of upper-triangular matrices. Let the respective Lie algebras be $\mathfrak{g}$ and $\mathfrak{b}$. The flag variety is the homogenous space $G / B$. It is known to be a smooth complex projective variety $[\bar{H}$, Section 21]. Hessenberg varieties are a family of subvarieties of the flag variety parametrized by an element $\mathbf{X} \in \mathfrak{g}$ and a function $h:\{1,2, \cdots, n\} \longmapsto\{1,2, \cdots, n\}$ [dMPS],[T2].

Hessenberg varieties are the space of ordered bases which represent $X$ in a form (i.e. Hessenberg form) under which numerical algorithms can be efficiently performed [dMPS]. Hessenberg varieties have also found applications in other fields, including combinatorics, geometry, and representation theory. Wellknown examples include the flag variety, the toric variety associated to the Coxeter complex and the Springer variety $[\overline{\mathrm{P}}],[\mathrm{Sp}],[\mathrm{St}],[\mathrm{T} 3]$.

When $\mathrm{X}$ is semisimple with distinct eigenvalues (i.e. regular semisimple) the Hessenberg varieties are smooth [dMPS, Theorem 6]. In this case, we construct the equivariant cohomology combinatorially using GKM theory [GKM],[KT],[T1]. This approach presents the equivariant cohomology using a combinatorial graph. These graphs are subgraphs of the Bruhat graph (see Figure 3 for examples).

Definition 2.1 An $h$-function is a non-decreasing function $h:\{1,2, \cdots, n\} \longmapsto\{1,2, \cdots, n\}$ such that $h(i) \geq i$ for each $i$. Let $E_{i, j}$ be the $n \times n$ matrix which is one in entry $\{i, j\}$ and zero elsewhere. A Hessenberg space is the complex vector space spanned by the $E_{i, j}$ such that $h(j) \geq i$ for each pair $i, j$. Hessenberg spaces will be denoted $H_{h}$ and $h$-functions $h=h(1) h(2) \cdots h(n)$.

Example 2.2 We write $H_{h}$ as the set of matrices with $*$ 's in positions $\{i, j\}$ such that $h(j) \geq i$ and 0 in the other positions. For example, let $h(i)=i+1$ for $i=1,2, \cdots, n-1$. Then $H_{h}$ is the complex span of the matrices $E_{i, j}$ where $i \leq j+1$. 


$$
H_{23455}=\left\{\left(\begin{array}{ccccc}
* & * & * & * & * \\
* & * & * & * & * \\
0 & * & * & * & * \\
0 & 0 & * & * & * \\
0 & 0 & 0 & * & *
\end{array}\right)\right\}
$$

Fig. 1: A Hessenberg space.

Definition 2.3 Fix $\mathrm{X} \in \mathfrak{g}$ and an $h$-function. A Hessenberg variety is the subvariety of $G / B$ given by

$$
\mathrm{X}_{h}:=\left\{g B \in G / B \mid g^{-1} \mathbf{X} g \in H_{h}\right\} .
$$

This is a closed set in $G / B$ and hence a projective variety.

Example 2.4 Examples of Hessenberg varieties.

(1.) When $h(i)=n$ for all $i$, then $H_{h}=\mathfrak{g}$. For any $\mathrm{X} \in \mathfrak{g}$ the Hessenberg variety $\mathrm{X}_{h}$ is the flag variety $G / B$.

(2.) When $h(i)=i$ for all $i$, then $H_{h}=\mathfrak{b}$. When $X \in \mathfrak{g}$ is nilpotent the Hessenberg variety is the Springer variety $[$ Sp].

(3.) Consider the given by h-function, $h(i)=i+1$ for $i=1,2, \cdots, n-1$. When $\mathrm{X} \in \mathfrak{g}$ is regular semisimple (see Definition 3.5) the Hessenberg variety is the toric variety associated with the Coxeter complex of $S_{n}$ [dMPS. Theorem 11]. There is a symmetric group representation the cohomology of this variety induced by the action of $S_{n}$ on the root system. Procesi and Stembridge give two approaches to decomposing the cohomology into irreducible representations $[[P],[[S t]$. In Section 4 we study a generalization of this representation on other regular semisimple Hessenberg varieties.

\section{GKM Theory for regular semisimple Hessenberg varieties.}

Our goal is to study Hessenberg varieties via a representation of the symmetric group on the (ordinary and equivariant) cohomology with complex coefficients. Among the results we prove is that when the Hessenberg variety has multiple connected components the representation is a permutation representation.

We follow a combinatorial approach. We construct the equivariant cohomology using GKM theory $[\mathrm{GKM}]$. This gives a presentation of both the equivariant and ordinary cohomology of regular semisimple Hessenberg varieties in terms of the Bruhat order of the symmetric group.

This combinatorial viewpoint is a primary advantage of using GKM theory. The representation we will study uses equivariant cohomology in an essential way. In fact, it is not obvious that the ordinary homology carries an $S_{n}$-representation without the GKM approach (see Remark 4.6).

Here we introduce GKM theory as needed for our purposes. More thorough background can be found in either the source [GKM] or the expository article [T1]. For more examples, GKM theory has been used to calculate equivariant cohomology for the Grassmannians, Schubert varieties and the flag variety [KT],[T3],[T4]. 
Let $X$ be a smooth complex projective variety which carries an action of a complex algebraic torus $T$. GKM theory allows us to view $H_{T}^{*}(X, \mathbb{C})$, the equivariant cohomology of $X$, as a free module over $\mathbb{C}\left[t_{1}, t_{2}, \cdots t_{n}\right]$, the polynomial algebra over the Lie algebra of $T$.

In order to apply GKM theory $X$ must have finitely many of both the $T$-fixed points and one-dimensional $T$-orbits. Denote the fixed points by $X^{T}$ and the one-dimensional orbits by $\left\{\mathcal{O}_{1}, \mathcal{O}_{2}, \cdots, \mathcal{O}_{k}\right\}$. If $X$ satisfies these finiteness conditions, we call it a GKM space. In fact, the category of GKM spaces is larger than smooth category. All a GKM space must satisfy is a technical condition called equivariant formality [GKM, Section 1.2].

The fixed points and one dimensional orbits form a one-skeleton in $X$ relative to the $T$-action. We construct a combinatorial graph, called the moment graph, from this one-skeleton. The vertices are the fixed points and there is an edge between fixed points if they are the two fixed points in the closure of an orbit, $\mathcal{O}_{i}$. Each edge is labeled by $\alpha_{i}$, the $\mathbb{C}\left[t_{1}, \cdots t_{n}\right]$-annihilator of $\mathfrak{t}_{i}$, the Lie algebra of the point-wise stabilizer of $\mathcal{O}_{i}$. Further, we direct the edge from $x \stackrel{\alpha_{i}}{\longmapsto} y$ if and only if the torus acts on the tangent space $T_{x}\left(\overline{\mathcal{O}_{i}}\right)$ with weight $\alpha_{i}$ and on $T_{y}\left(\overline{O_{i}}\right)$ with weight $-\alpha_{i}$. [GKM Section 7.1].

Definition 3.1 Let $X$ be a GKM space. The moment graph of $X$ is the graph $\Gamma(X)=(\mathcal{V}, \mathcal{E})$ where the vertices are $\mathcal{V}=X^{T}$ and the labeled edges are

$$
\mathcal{E}=\left\{x \stackrel{\alpha_{i}}{\longmapsto} y \mid x, y \in \overline{\mathcal{O}_{i}} \cap X^{T} \text { and } \alpha_{i} \text { is the annihilator of } \mathfrak{t}_{i} \cdot\right\}
$$

All GKM spaces have a localization map $H_{T}^{*}(X, \mathbb{C}) \longrightarrow \mathbb{C}\left[t_{1}, \cdots, t_{n}\right]^{\oplus X^{T}}$ that is in fact injective. This is what permits the GKM presentation of the equivariant cohomology.

Theorem 3.2 (GKM presentation [GKM]) Let $X$ be a GKM space with moment graph $\Gamma(X)$. Then the equivariant cohomology of $X$ is given by

$$
H_{T}^{*}(X, \mathbb{C}):=\left\{p: X^{T} \longmapsto \mathbb{C}\left[t_{1}, \cdots, t_{n}\right] \mid \text { for } x \stackrel{\alpha_{i}}{\longmapsto} y, \text { the difference } p_{x}-p_{y} \in \alpha_{i}\right\} .
$$

The forgetful map which sets each $t_{i}=0$ relates the equivariant cohomology to the ordinary cohomology $H^{*}(X, \mathbb{C})$.

Proposition 3.3 There is a ring isomorphism

$$
H^{*}(X, \mathbb{C}) \cong \frac{H_{T}^{*}(X, \mathbb{C})}{\left\langle t_{1}, \cdots, t_{n}\right\rangle H_{T}^{*}(X, \mathbb{C})}
$$

One consequence is that any free $\mathbb{C}\left[t_{1}, \cdots, t_{n}\right]$-module basis for equivariant cohomology can be viewed as $\mathbb{C}$-vector space basis for ordinary cohomology by scalar restriction. Finally, the next result is helpful later (see Section 3.2).

Lemma 3.4 Let $X$ be GKM space with moment graph $\Gamma(X)$. Then the connected components of $X$ are GKM spaces whose moment graph the connected graph components of $\Gamma(X)$.

\subsection{Regular semisimple Hessenberg varieties.}

Here we give the GKM presentation for a family of Hessenberg varieties. Recall that $S_{n}$ embeds into $G$ as the subgroup of permutation matrices. This identification is key to exposing the connection between the geometry of the Hessenberg varieties and the combinatorics of the symmetric group. 
Definition 3.5 A semisimple element $\mathrm{X} \in \mathfrak{g}$ is regular when its eigenvalues are all distinct.

Fix a regular semisimple $X \in \mathfrak{g}$. In fact, because conjugation (change of basis) is an isomorphism of varieties we may assume $X$ is diagonal with distinct diagonal entries. De Mari-Procesi-Shayman proved that the Hessenberg varieties of regular semisimple $X$ are smooth [dMPS, Theorem 6]. Therefore, for $X_{h}$ to be a GKM space we only need an appropriate torus action.

Let $T$ be the subgroup of diagonal matrices in $G$. The action of $T$ on $G / B$ given by $t \cdot g B=\operatorname{tg} B$ restricts to $\mathrm{X}_{h}$ because $T=C_{G}(\mathrm{X})$. With respect to this torus action $G / B$ is a GKM space, and so is $\mathrm{X}_{h}$.

Definition 3.6 Let $w \in S_{n}$. The inversions of $w$ is the set $\operatorname{inv}(v):=\left\{i<j \mid w^{-1}(i)>w^{-1}(j)\right\}$.

Proposition 3.7 Every regular semisimple Hessenberg variety is a GKM space, with moment graph $\Gamma\left(\mathrm{X}_{h}\right)=(\mathcal{V}, \mathcal{E})$ given by:

$$
\begin{aligned}
& \mathcal{V}:=\left\{w B \mid w \in S_{n}\right\} \\
& \mathcal{E}:=\left\{w^{\prime} B \stackrel{t_{i}-t_{j}}{\longmapsto} w B \mid w^{\prime}=(i j) w, i<j \in \operatorname{inv}(w) \text { and } w^{-1}(i) \leq h\left(w^{-1}(j)\right)\right\}
\end{aligned}
$$

Furthermore, the equivariant cohomology is

$$
H_{T}^{*}\left(\mathbf{X}_{h}, \mathbb{C}\right):=\left\{p: S_{n} \longmapsto \mathbb{C}\left[t_{1}, \cdots, t_{n}\right] \mid \text { for } w^{\prime} B \stackrel{t_{i}-t_{j}}{\longmapsto} w B \text { the difference } p_{w}-p_{w^{\prime}} \in\left\langle t_{i}-t_{j}\right\rangle\right\} .
$$

Proof outline: J. Carrell proved the moment graph for $G / B$ is as above for the function $h(i)=n$ for all $i[\mathrm{C}]$. Any regular semisimple Hessenberg variety $\mathrm{X}_{h}$ carries the same torus action as $G / B$. Therefore the moment graph of $\mathrm{X}_{h}$ is a subgraph of that of $G / B$. It is then a direct calculation to show which orbits from $G / B$ are contained in $\mathrm{X}_{h}$.

Example 3.8 To determine whether a tuple of polynomials is a class one must check that the difference of polynomials at adjacent vertices are multiples of the $t_{i}-t_{j}$. Figure 2 provides an example in $\left.H_{T}^{*}\left(\mathrm{X}_{223}, \mathbb{C}\right)\right)$, where one is a class and the other not.

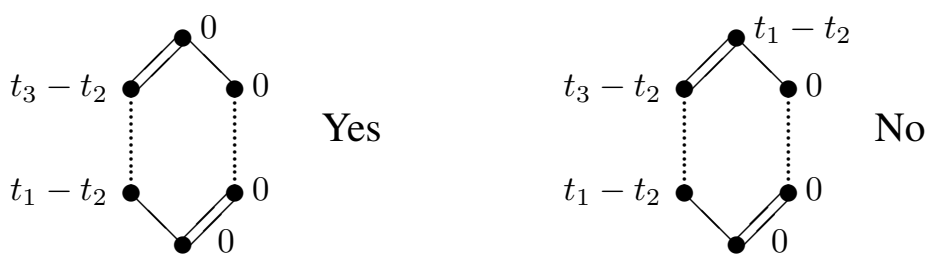

Fig. 2: Examples of the equivariant condition.

The relation $w^{\prime} B \stackrel{t_{i}-t_{j}}{\longmapsto} w B$ if $i<j \in \operatorname{inv}(w)$ is exactly the condition defining the cover relation for the Bruhat order on the symmetric group [BB, Section 2.1]. When the Hessenberg variety is the flag variety the moment graph is a labeled Bruhat graph. Hence, the moment graph of any regular semisimple Hessenberg variety is a labeled subgraph of the Bruhat graph. This fact is the underpinning of the combinatorics of this paper. Because of it we are able to use facts about the Bruhat order, parabolic subgroups, and minimal coset representatives as we study these varieties. 
Frequently, we will suppress the coset notation and write edges as $w^{\prime} \stackrel{t_{i}-t_{j}}{\longmapsto} w$. The conditions (Proposition 3.7 defining an edge are cumbersome. This can remedied by calculating a right-hand version of the edge condition.

Corollary 3.9 There is an edge between $w$ and $w^{\prime}$ if and only if $w^{\prime}=w\left(i^{\prime} j^{\prime}\right)$ for $i^{\prime}<j^{\prime}$ and $h\left(i^{\prime}\right) \geq j^{\prime}$.

Proof: If $w^{\prime} \stackrel{t_{i}-t_{j}}{\longmapsto} w$ is an edge then $(i j) w=w w^{-1}(i j) w=w\left(w^{-1}(j) w^{-1}(i)\right)$. Direct calculation shows $i^{\prime}=w^{-1}(j)<w^{-1}(i)=j^{\prime}$ satisfies the condition.

This right-hand condition makes it easier to construct the moment graph, but we must still use the lefthand version to calculate the classes $p \in H_{T}^{*}\left(\mathrm{X}_{h}, \mathbb{C}\right)$. The transpositions $\left(i^{\prime} j^{\prime}\right)$ satisfying the conditions of this corollary will be called right-transpositions.

Example 3.10 For $G L_{3}(\mathbb{C})$, up to homeomorphism, there are four regular semisimple Hessenberg varieties. In this case, we can identify these varieties. When $h=123$, the variety is the fixed point set of the torus. For $h=223$, the variety is three disjoint copies of $\mathbb{C P}^{1}$. For $h=233$, the variety is the toric variety associated with the decomposition of the Coxeter complex (see also Example 2.4). Lastly, $h=333$ is the flag variety $G L_{3}(\mathbb{C}) / B$ (see also Example 2.4 ).

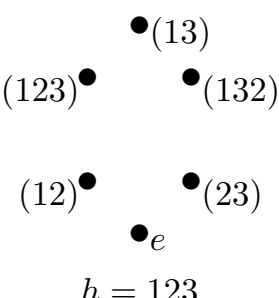

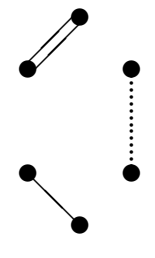

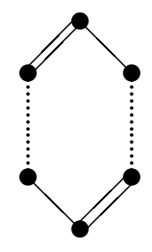

$h=223$

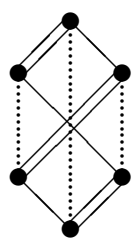

$h=333$

Fig. 3: Moment graphs of regular semisimple Hessenberg varieties.

\subsection{Disconnected regular semisimple Hessenberg varieties.}

In this section we give a criterion for when $X_{h}$ is disconnected and give an explicit decomposition of $X_{h}$ into homeomorphic connected components. Fix a regular semisimple element $\mathrm{X}$ and an $h$-function $h$. For the rest of the paper, all Hessenberg varieties will be regular semisimple.

Definition 3.11 The parabolic subgroup of $\mathrm{X}_{h}$ is $W_{h}:=\left\langle(i j) \in S_{n} \mid h(i) \geq j\right\rangle$.

The name parabolic subgroup comes from the theory of Coxeter groups. In fact, $W_{h}$ is generated by the simple transpositions $(i i+1)$ such that $h(i) \geq i+1$, and so is a parabolic subgroup of the Coxeter group $S_{n}$. These subgroups also arise in the representation theory of the symmetric group, where they are called Young subgroups. It will be important to know that up to isomorphism, these subgroups have the form $S_{\lambda}=S_{\lambda_{1}} \times \cdots \times S_{\lambda_{k}}$ for $\lambda=\left(\lambda_{1}, \lambda_{2}, \cdots, \lambda_{k}\right)$ a partition of $n$,

Since the parabolic subgroup is generated by the simple transposition satisfying the right-hand condition they do not uniquely determine the Hessenberg variety. For example, the Hessenberg varieties $\mathrm{X}_{2334}$ and $\mathrm{X}_{3334}$ both have parabolic subgroup isomorphic to $S_{(3,1)}$. Despite this parabolic subgroups are useful when describing the moment graph. 
Every permutation $u \in W_{h}$ can be written as a product of right-transpositions. In terms of the moment graph, this product corresponds to a path between the identity and $u$. Hence, this subgroup generates the graph component containing the identity. By Lemma 3.4 this graph component corresponds to another GKM space. We will call this space the identity component and denote it $\mathrm{X}_{h}^{\circ}$.

Lemma 3.12 Fix an $h$-function $h$. There are $\left[S_{n}: W_{h}\right]$ connected components of regular semisimple Hessenberg variety.

Proof: From Lemma 3.4 we know it is sufficient to count the graph components of the moment graph. Now by Corollary 3.9 , the permutations $u, v \in S_{n}$ are in the same graph component if and only if there is a $w \in W_{h}$ such that $u=v w$. This is equivalent to $u W_{h}=v W_{h}$. Hence, there are $\left[S_{n}: W_{h}\right]$ connected components of $\mathrm{X}_{h}$.

This lemma shows that the components of the moment graph respect the right multiplication structure of $W_{h}$. Hence, the moment graph is composed of isomorphic graph components indexed by the left cosets of $W_{h}$. This combinatorial property hints that when the Hessenberg variety is disconnected then connected components are homeomorphic. This is true.

Proposition 3.13 For a disconnected Hessenberg variety, $\mathrm{X}_{h}^{\circ} \cong \prod_{i=1}^{k} \mathrm{X}_{h}^{\lambda_{i}}$, where the $\mathrm{X}_{h}^{\lambda_{i}}$ are regular semisimple Hessenberg varieties in $G L_{\lambda_{i}}(\mathbb{C})$.

Proof outline: Suppose $W_{h} \cong S_{\lambda}$ for $\lambda=\left(\lambda_{1}, \cdots, \lambda_{k}\right)$ a partition of $n$. For $g B \in \mathrm{X}_{h}^{\circ}$ the product $g^{-1} \mathrm{X} g$ is mapped to the subspace of $H_{h}$ consisting of block diagonal matrices with dimensions given by $\lambda$. This gives $k$ independent conditions each of which describes a Hessenberg variety in $G L_{\lambda_{i}}(\mathbb{C})$.

Corollary 3.14 Let $\mathrm{X}_{h}$ be a disconnected regular semisimple Hessenberg variety. Then the connected components of $\mathrm{X}_{h}$ are all homeomorphic.

Proof outline: Let $\mathcal{J}$ be a connected component of $\mathrm{X}_{h}$ and pick $u \in \mathcal{J}^{T}$. Consider the map given by left translation by $u^{-1}$. This maps $\mathcal{J}$ homeomorphically onto $\left(u^{-1} \mathrm{X} u\right)_{h}^{\circ}$ i.e. the identity component of the Hessenberg variety corresponding to the regular semisimple element $u^{-1} \mathrm{X} u$ and the same $h$-function $h$. By Proposition 3.13, $\mathcal{J}$ is homeomorphic to $\mathrm{X}_{h}^{\circ}$.

\section{A representation of the symmetric group.}

In this section we define a representation of the symmetric group on the equivariant cohomology of regular semisimple Hessenberg varieties. Geometrically this representation is defined from an action of $S_{n}$ on the the moment graph. Here we review necessary background on the representation theory of the symmetric group. A classic source for these results is [JK].

The representation ring of $S_{n}$ has two free $\mathbb{Z}$-bases, both parameterized by partitions of $n$. The first basis is the collection of irreducible representations $V^{\lambda}$ with characters $\chi^{\lambda}$. The second basis consists of permutation representations $P^{\lambda}$ with character $\psi^{\lambda}$. These are obtained from the left multiplication action of $S_{n}$ on the cosets of $S_{\lambda}$, i.e. the cosets of Young subgroups. Equivalently, each $P^{\lambda}$ is constructed by inducing the trivial representation of $S_{\lambda}$ to $S_{n}$. We will be interested in decomposing the $P^{\mu}$ in terms of the $V^{\lambda}$. 
Definition 4.1 The lexicographic order on partitions of $n$ is given by

$$
\lambda>\mu \text { if the first non-vanishing } \lambda_{i}-\mu_{i} \text { is positive. }
$$

Definition 4.2 The Kostka numbers $K_{\mu \lambda}$ are the number of semistandard Young tableaux of shape $\mu$ and weight $\lambda$.

Consider the matrix with Kostka numbers as entries. If we order the rows and columns (i.e. partitions) in lexicographic order we obtain a transition matrix between permutation representations and irreducible representations. This is classically known as Young's Rule.

Proposition 4.3 (Young's Rule [JK]]) Let $\tau^{\lambda}$ denote the character of the trivial representation for the Young subgroup $S_{\lambda}$. Then the induced character $\operatorname{Ind}_{S_{\lambda}}^{S_{n}} \tau^{\lambda}$ is given by

$$
\operatorname{Ind}_{S_{\lambda}}^{S_{n}} \tau^{\lambda}:=\psi^{\lambda}=\chi^{\lambda}+\sum_{\mu>\lambda} K_{\mu \lambda} \chi^{\mu}
$$

\subsection{The representation on the cohomology.}

The symmetric group acts on $\mathbb{C}\left[t_{1}, \cdots, t_{n}\right]$ by permuting variables. That is for $w \in S_{n}$ and a polynomial $f\left(t_{1}, \cdots, t_{n}\right)$, the action of $w$ on $f\left(t_{1}, \cdots, t_{n}\right)$ is given by

$$
w * f\left(t_{1}, t_{2}, \cdots, t_{n}\right)=f\left(t_{w(1)}, t_{w(2)}, \cdots, t_{w(n)}\right) .
$$

This action is a ring automorphism of $\mathbb{C}\left[t_{1}, \cdots, t_{n}\right]$. We can extend this to a representation of $S_{n}$ on $H_{T}^{*}\left(\mathrm{X}_{h}\right)$.

Proposition 4.4 Let $\mathrm{X}_{h}$ be a regular semisimple Hessenberg variety. There is a representation of $S_{n}$ on $H_{T}^{*}\left(\mathrm{X}_{h}, \mathbb{C}\right)$ given by

$$
(w \cdot p)_{u}=w * p_{w^{-1} u} .
$$

Further, using the isomorphism of Proposition 3.3 this is a representation on $H^{*}\left(\mathrm{X}_{h}, \mathbb{C}\right)$.

We defer the proof until after the next example. This action is easiest understood when $w=(i j)$ is a transposition. In this case, the action of $(i j)$ interchanges the polynomials across edges in the moment graph for $G / B$ labeled $t_{i}-t_{j}$, and permutes the variables. For example Figure 4 shows the action of $(12)$ on a class in $H_{T}^{*}\left(\mathrm{X}_{233}, \mathbb{C}\right)$.
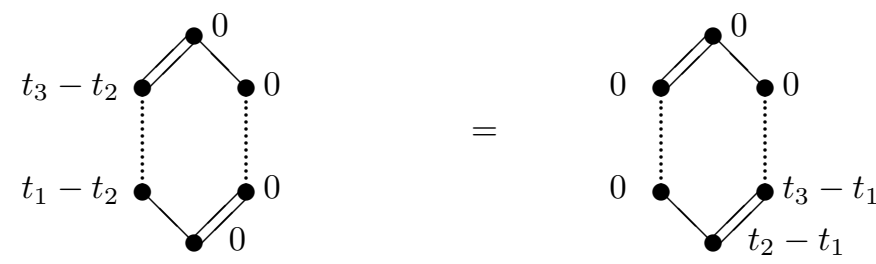

Fig. 4: The action on an equivariant class.

The next Lemma shows that $S_{n}$ acts on the moment graph of regular semisimple Hessenberg varieties. It is key to proving Proposition 4.4 
Lemma 4.5 Let $v \in S_{n}$ and $w^{\prime} \stackrel{t_{i}-t_{j}}{\longmapsto} w$ be an edge in the moment graph. The map $\varphi_{v}: S_{n} \rightarrow S_{n}$ defined by $\varphi_{v}(w)=v^{-1} w$ sends the edge $w^{\prime} \stackrel{t_{i}-t_{j}}{\longmapsto} w$ to

- $v^{-1} w^{\prime} \longmapsto v^{-1} w$ with label $t_{v^{-1}(i)}-t_{v^{-1}(j)}$ if $i<j \notin \operatorname{inv}(v)$.

- $v^{-1} w \longmapsto v^{-1} w^{\prime}$ with label $t_{v^{-1}(j)}-t_{v^{-1}(j)}$ if $i<j \in \operatorname{inv}(v)$.

Proof: The proof in both cases is similar. We prove it when $i<j \in \operatorname{inv}(v)$. We have

$$
v^{-1} w=v^{-1}(i j) w^{\prime}=\left(v^{-1}(i) v^{-1}(j)\right) v^{-1} w^{\prime}
$$

and $v^{-1}(i)>v^{-1}(j)$. Therefore, we must check that $v^{-1}(j)<v^{-1}(i) \in \operatorname{inv}\left(v^{-1} w^{\prime}\right)$ and that

$$
\left(v^{-1} w^{\prime}\right)^{-1}\left(v^{-1}(j)\right) \leq h\left(\left(v^{-1} w^{\prime}\right)^{-1}\left(v^{-1}(i)\right)\right) .
$$

This follows directly from the relation $\left(v^{-1} w^{\prime}\right)^{-1}\left(v^{-1}(i)\right)=w^{-1}(j)$ and $\left(v^{-1} w^{\prime}\right)^{-1}\left(v^{-1}(j)\right)=w^{-1}(i)$.

Proof of Proposition 4.4; Let $u^{\prime} \stackrel{t_{i}-t_{j}}{\longmapsto} u$ be an edge and $p \in H_{T}^{*}\left(\mathbf{X}_{h}, \mathbb{C}\right)$. We must show that $w \cdot p$ satisfies the equivariant condition, i.e. $(w \cdot p)_{u}-(w \cdot p)_{u^{\prime}} \in\left\langle t_{i}-t_{j}\right\rangle$. This follows from the action on the moment graph

$$
(w \cdot p)_{u}-(w \cdot p)_{v}=w *\left(p_{w^{-1}(u)}-p_{w^{-1}(v)}\right) \in w *\left\langle t_{w^{-1}(i)}-t_{w^{-1}(j)}\right\rangle=\left\langle t_{i}-t_{j}\right\rangle .
$$

The second claim is immediate.

Remark 4.6 In the case of $G / B$, the group $S_{n}$ acts on all of $G / B$ by left multiplication. Therefore, the representation on the cohomology is defined geometrically by this action. This is not the case for general Hessenberg varieties. For example, if $h=233$ consider the matrices

$$
\mathbf{X}=\left(\begin{array}{lll}
1 & 0 & 0 \\
0 & 2 & 0 \\
0 & 0 & 3
\end{array}\right) \quad g=\left(\begin{array}{lll}
1 & 1 & 1 \\
2 & 1 & 0 \\
1 & 0 & 0
\end{array}\right) \quad w_{(12)}=\left(\begin{array}{lll}
0 & 1 & 0 \\
1 & 0 & 0 \\
0 & 0 & 1
\end{array}\right)
$$

Direct calculation using Definition 2.3 gives

$$
g^{-1} \times g=\left(\begin{array}{ccc}
3 & 0 & 0 \\
-2 & 2 & 0 \\
0 & -1 & 1
\end{array}\right) \quad\left(w_{(12)} g\right)^{-1} \times\left(w_{(12)} g\right)=\left(\begin{array}{ccc}
3 & 0 & 0 \\
-4 & 1 & 0 \\
3 & 1 & 2
\end{array}\right) \text {. }
$$

This means $g B \in \mathrm{X}_{233}$ while $w_{(12)} \cdot g B \notin \mathrm{X}_{233}$. In other words, this Hessenberg variety is not invariant under the left multiplication action of $S_{n}$, only its moment graph is. For this reason, the representation will vary as the moment graph varies, so the combinatorial approach GKM theory provides is valuable when studying this representation.

Tymoczko studied the representation on $G / B$ using the same GKM approach we use here. She obtained a combinatorial proof that the representation on ordinary cohomology is trivial [T4]. This result is known in the literature, but the proofs rely on geometric arguments.

Theorem 4.7 (Tymoczko [T4]) The representation on $H^{*}(G / B, \mathbb{C})$ decomposes into $\left|S_{n}\right|$ copies of the trivial representation. 


\subsection{The representation on disconnected Hessenberg varieties.}

Let $w_{i}, \cdots, w_{k}$ be the system of coset representatives of $W_{h}$ minimal length [BB, Section 2.4]. Proposition 3.14 allows us to write $\mathrm{X}_{h}$ as the disjoint union of the translates $w_{i} \mathrm{X}_{h}^{\circ}$. Hence the equivariant cohomology is:

$$
H_{T}^{*}\left(\mathbf{X}_{h}, \mathbb{C}\right)=\bigoplus_{w_{i}} H_{T}^{*}\left(w_{i} \mathbf{X}_{h}^{\circ}, \mathbb{C}\right) .
$$

Next we determine an explicit isomorphism between $H_{T}^{*}\left(\mathrm{X}_{h}^{\circ}, \mathbb{C}\right)$ and $H_{T}^{*}\left(w_{i} \mathrm{X}_{h}^{\circ}, \mathbb{C}\right)$. This will be key to showing $H_{T}^{*}\left(\mathrm{X}_{h}, \mathbb{C}\right)$ is the induced representation of $H_{T}^{*}\left(\mathrm{X}_{h}^{\circ}, \mathbb{C}\right)$ through $W_{h}$.

Proposition 4.8 There is an isomorphism given by $\varphi_{w_{i}}: H_{T}^{*}\left(\mathrm{X}_{h}^{\circ}, \mathbb{C}\right) \rightarrow H_{T}^{*}\left(w_{i} \mathrm{X}_{h}^{\circ}, \mathbb{C}\right)$ defined by

$$
p_{u} \longmapsto p_{w_{i} u}:=w_{i} * p_{u} .
$$

Proof outline: This is a direct computation using the same argument as Proposition 4.4

With this we have descriptions of the variety $\mathrm{X}_{h}$, the moment graph $\Gamma\left(\mathrm{X}_{h}\right)$, and the equivariant cohomology $H_{T}^{*}\left(\mathrm{X}_{h}, \mathbb{C}\right)$ in terms of the analogs for the identity component $\mathrm{X}_{h}^{\circ}$. Further, from Proposition 3.13 and the the Künneth formula we have

$$
H_{T}^{*}\left(\mathrm{X}_{h}^{\circ}, \mathbb{C}\right) \cong H_{T}^{*}\left(\prod_{i=1}^{k} \mathrm{X}_{h}^{\lambda_{i}}, \mathbb{C}\right) \cong \bigotimes_{i=1}^{k} H_{T}^{*}\left(\mathrm{X}_{h}^{\lambda_{i}}, \mathbb{C}\right) .
$$

Lemma 4.9 The equivariant cohomology of $\mathrm{X}_{h}^{\circ}$ is a representation of $W_{h}$.

Proof: Let $W_{h} \cong S_{\lambda}=S_{\lambda_{1}} \times \cdots \times S_{\lambda_{k}}$. From Proposition 3.13 and Equation 2 we define the representation on $H_{T}^{*}\left(\mathrm{X}_{h}^{\circ}, \mathbb{C}\right)$ component-wise.

This leads to the first main theorem.

Theorem 4.10 Let $\mathrm{X}_{h}$ be a disconnected Hessenberg variety with parabolic subgroup $W_{h}$. Then as representations $H_{T}^{*}\left(\mathrm{X}_{h}\right)=\operatorname{Ind}_{W_{h}}^{S_{n}} H_{T}^{*}\left(\mathrm{X}_{h}^{\circ}, \mathbb{C}\right)$.

Proof: Proposition 3.14 gives that

$$
H_{T}^{*}\left(\mathrm{X}_{h}, \mathbb{C}\right)=\bigoplus_{w_{i} \text { coset reps }} H_{T}^{*}\left(w_{i} \mathrm{X}_{h}^{\circ}, \mathbb{C}\right),
$$

and by Lemma $4.9 H_{T}^{*}\left(\mathrm{X}_{h}^{\circ}, \mathbb{C}\right)$ is $W_{h}$-stable. It follows from Proposition 4.8 and Equation 1 that each $p \in H_{T}^{*}\left(\mathbf{X}_{h}, \mathbb{C}\right)$ is uniquely expressed as $p=\sum_{w_{i}} w_{i} * p^{i}$ for some $p^{i} \in H_{T}^{*}\left(\mathbf{X}_{h}^{\circ}, \mathbb{C}\right)$. By definition $H_{T}^{*}\left(\mathrm{X}_{h}, \mathbb{C}\right)$ is the induced representation $\operatorname{Ind}_{W_{h}}^{S_{n}} H_{T}^{*}\left(\mathrm{X}_{h}^{\circ}, \mathbb{C}\right)$

This result permits us to decompose the ordinary cohomology into irreducible representations when the Hessenberg variety is parabolic.

Definition 4.11 Whenever the Hessenberg space $H_{h}$ is a parabolic subalgebra of $\mathfrak{g}$ we call the Hessenberg variety parabolic. 


$$
H_{3334}=\left\{\left(\begin{array}{cccc}
* & * & * & * \\
* & * & * & * \\
* & * & * & * \\
0 & 0 & 0 & *
\end{array}\right)\right\} \quad H_{2334}=\left\{\left(\begin{array}{cccc}
* & * & * & * \\
* & * & * & * \\
0 & * & * & * \\
0 & 0 & 0 & *
\end{array}\right)\right\}
$$

Fig. 5: A parabolic Hessenberg space and a non-parabolic Hessenberg space.

In other words, a Hessenberg variety is parabolic whenever $H_{h}$ "forms a block-staircase" in $\mathfrak{g}$. The size of the blocks correspond to the parts of $\lambda$ in $W_{h} \cong S_{\lambda_{1}} \times \cdots \times S_{\lambda_{k}}$.

Example 4.12 Compare $H_{3334}$ which is a parabolic Hessenberg, and $H_{2334}$ which is not. They have both have parabolic subgroup isomorphic to $S_{(3,1)}$, but $E_{3,1} \notin H_{2334}$ (see Figure 5).

Proposition 4.13 The identity component of a parabolic Hessenberg is homeomorphic to the product $G L_{\lambda_{1}}(\mathbb{C}) / B_{\lambda_{1}} \times \cdots \times G L_{\lambda_{k}}(\mathbb{C}) / B_{\lambda_{k}}$, where $\lambda=\left(\lambda_{1}, \cdots, \lambda_{k}\right)$ is the partition corresponding to the group $W_{h} \cong S_{\lambda}$.

Proof: Use Proposition 3.13 and check that each factor in the product is isomorphic to a flag variety.

Let $\chi^{(n)}$ be the character of the trivial representation of $S_{n}$ so $\tau^{\lambda}=\chi^{\left(\lambda_{1}\right)} \times \cdots \times \chi^{\left(\lambda_{k}\right)}$ is the trivial character of $S_{\lambda}$. As a corollary we obtain the following.

Corollary 4.14 Let $\mathrm{X}_{h}^{\circ}$ be the identity component of a parabolic Hessenberg. Then the $W_{h}$-representation on $H^{*}\left(\mathrm{X}_{h}^{\circ}, \mathbb{C}\right)$ is trivial and has $\left|W_{h}\right| \tau^{\lambda}$ as its character.

Proof: The proposition gives $\mathrm{X}_{h}^{\circ} \cong G L_{\lambda_{1}}(\mathbb{C}) / B_{\lambda_{1}} \times \cdots \times G L_{\lambda_{k}}(\mathbb{C}) / B_{\lambda_{k}}$. From Tymoczko's result (Theorem 4.7) and Lemma 4.9 the character is

$$
\left|S_{\lambda_{1}}\right| \chi^{\left(\lambda_{1}\right)} \times \cdots \times\left|S_{\lambda_{k}}\right| \chi^{\left(\lambda_{k}\right)}=\left(\prod_{i=1}^{k}\left|S_{\lambda_{i}}\right|\right) \chi^{\left(\lambda_{1}\right)} \times \cdots \times \chi^{\left(\lambda_{k}\right)}=\left|W_{h}\right| \tau^{\lambda} .
$$

Finally, we obtain our main result. From Theorem 4.10 together with Corollary 4.14 we have that $H^{*}\left(\mathrm{X}_{h}, \mathbb{C}\right)=\left|W_{h}\right| P^{\lambda}$, the permutation representation associated to $W_{h} \cong S_{\lambda}$. Using Young's rule we obtain the irreducible decomposition of the ordinary cohomology for all parabolic regular semisimple Hessenberg varieties.

Theorem 4.15 Let $\mathrm{X}_{h}$ be a parabolic regular semisimple Hessenberg variety, with parabolic subgroup $W_{h} \cong S_{\lambda}$. The character of the representation $\chi^{h}$ decomposes in ordinary cohomology as

$$
\chi^{h}=\left|W_{h}\right| \chi^{\lambda}+\sum_{\mu>\lambda}\left|W_{h}\right| K_{\mu \lambda} \chi^{\mu} .
$$

Proof: We know $H^{*}\left(\mathrm{X}_{h}, \mathbb{C}\right)=\operatorname{Ind}_{W_{h}}^{S_{n}} H^{*}\left(\mathrm{X}_{h}^{\circ}, \mathbb{C}\right)$. For parabolic $\mathrm{X}_{h}$ the character on the identity component is $\left|W_{h}\right| \tau^{\lambda}$ (see Corollary 4.14). Young's Rule gives the result. 


\section{References}

[BB]

$[\mathrm{C}]$

[dMPS]

$[\mathrm{GKM}]$

$[\mathrm{JK}]$

$[\mathrm{P}]$

$[\mathrm{Sp}]$

A. Bjorner and F. Brenti, Combinatorics of Coxeter Groups, Springer, New York, 2005.

J. Carrell, The Bruhat graph of a Coxeter group, a conjecture of Deodhar, and rational smoothness of Schubert varieties, Algebraic groups and their generalizations: Classical methods, 53-61, Proc. Symp. Pure Math. 56 (1994), Amer. Math. Soc. Providence, RI, 1994.

F. de Mari, C. Procesi, and M. Shayman, Hessenberg varieties, Trans. Amer. Math. Soc. 332 (1992), 529-534.

M. Goresky, R. Kottwitz, and R. MacPherson, Equivariant cohomology, Koszul duality, and the localization theorem, Invent. Math. 131 (1998), 25-83.

J. Humphreys, Linear Algebraic Groups, Springer, New York, 1975.

G. James and A. Kerber, The Representation Theory of the Symmetric Group, Addison-Wesley, Reading, MA, 1981.

A. Knutson and T. Tao, Puzzles and (equivariant) cohomology of Grassmannians, Duke Math. J. 119 (2003), 221-260.

C. Procesi, The toric variety associated to Weyl chambers, Mots, 153-161, Lang. Raison. Calc. Hermès, Paris, 1990.

T. Springer, A Construction of Representations of Weyl Groups, Invent. Math. 44 (1978), 279-293.

J. Stembridge, Eulerian numbers, tableaux, and the Betti numbers of a toric variety, Discrete Math. 99 (1992), 307-320.

J. Tymoczko, An introduction to equivariant cohomology and homology, following Goresky, Kottwitz, and MacPherson, Snowbird lectures in algebraic geometry, 169188, Con. Math. 388, Amer. Math. Soc., Providence, RI, 2005.

J. Tymoczko, Linear conditions imposed on flag varieties, Amer. J. Math. 128 (2006), 1587-1604.

J. Tymoczko, Permutation actions on Equivariant cohomology, Toric topology, 365384, Con. Math. 460, Amer. Math. Soc., Providence, RI, 2008.

J. Tymoczko, Permutation representations on Schubert varieties, Amer. J. Math. 130 (2008), no. 5, 1171-1194. 